\title{
BMJ Open WHISPER or SHOUT study: protocol of a cluster-randomised controlled trial assessing mHealth sexual reproductive health and nutrition interventions among female sex workers in Mombasa, Kenya
}

To cite: Ampt FH, Mudogo C, Gichangi $P$, et al. WHISPER or SHOUT study: protocol of a cluster-randomised controlled trial assessing mHealth sexual reproductive health and nutrition interventions among female sex workers in Mombasa, Kenya. BMJ Open 2017;7:e017388. doi:10.1136/ bmjopen-2017-017388

- Prepublication history for this paper is available online. To view these files please visit the journal online (http://dx.doi org/10.1136/bmjopen-2017017388).

FHA and CM contributed equally.

Received 20 April 2017

Revised 23 June 2017

Accepted 30 June 2017

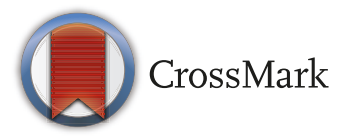

For numbered affiliations see end of article.

Correspondence to

Professor Stanley Luchters;

stanley.luchters@burnet.edu.au

\section{ABSTRACT}

Introduction New interventions are required to reduce unintended pregnancies among female sex workers (FSWs) in low- and middle-income countries and to improve their nutritional health. Given sex workers' high mobile phone usage, repeated exposure to short messaging service (SMS) messages could address individual and interpersonal barriers to contraceptive uptake and better nutrition.

Methods In this two-arm cluster randomised trial, each arm constitutes an equal-attention control group for the other. SMS messages were developed systematically, participatory and theory-driven and cover either sexual and reproductive health (WHISPER) or nutrition (SHOUT). Messages are sent to participants 2-3 times/week for 12 months and include fact-based and motivational content as well as role model stories. Participants can send reply texts to obtain additional information. Sex work venues (clusters) in Mombasa, Kenya, were randomly sampled with a probability proportionate to venue size. Up to 10 women were recruited from each venue to enrol 860 women. FSWs aged 16-35 years, who owned a mobile phone and were not pregnant at enrolment were eligible. Structured questionnaires, pregnancy tests, HIV and syphilis rapid tests and full blood counts were performed at enrolment, with subsequent visits at 6 and 12 months. Analysis The primary outcomes of WHISPER and SHOUT are unintended pregnancy incidence and prevalence of anaemia at 12 months, respectively. Each will be compared between study groups using discrete-time survival analysis.

Potential limitations Contamination may occur if participants discuss their intervention with those in the other trial arm. This is mitigated by cluster recruitment and only sampling a small proportion of sex work venues from the sampling frame.

Conclusions The design allows for the simultaneous testing of two independent mHealth interventions for which messaging frequency and study procedures are identical. This trial may guide future mHealth initiatives and provide methodological insights into use of reciprocal control groups.

Trial registration number ACTRN12616000852459; Preresults.

\section{INTRODUCTION}

\section{Female sex work}

Despite sex work being very common in sub-Saharan Africa, with 1 in 20 women estimated to have exchanged sex for money, goods or other favours, ${ }^{1}$ it remains a highly stigmatised and mostly criminalised practice, including in Kenya. ${ }^{2}$ The hostile politico-legal and social environment limits sex workers' access to health services, especially for sexual and reproductive health (SRH) and for preventing and treating HIV and other sexually transmitted infections (STIs). ${ }^{3}$ Strategies such as providing peer education and outreach services are often unable to overcome the myriad structural, personal and financial challenges that female sex workers (FSWs) face. ${ }^{4}$ This complex situation calls for new interventions that complement the current package of services and improve health and social outcomes for sex workers.

Unintended pregnancies among female sex workers In many low-income countries, increased provision of low-cost contraceptive methods has raised contraception coverage in the general population, ${ }^{56}$ but such gains remain inequitably distributed within countries, ${ }^{7}$ and they have not translated into improvements 


\section{Strengths and limitations of this study}

- Use of a cluster-RCT with reciprocal controls allows for the simultaneous testing of two mHealth interventions, for which message frequency and study processes are identical; only the content of the messages differs. The design mitigates biases from unequal attention in trial groups, positive expectations and nonblinding. Moreover, the approach is efficient, using considerably fewer resources than two independent trials.

- Contamination may occur if women receiving SRH messages share them or discuss their content with women in the nutrition arm, and vice versa. Contamination would diminish the ability to detect a difference in effectiveness between the two groups. Cluster-based intervention allocation could limit this, though FSWs often work from multiple venues. In addition, we recruited only a small proportion of sex workers across a large geographic area (up to 106 of 760 hot spots, and 860 of an estimated 8,516 FSWs), which diminishes the chances of interaction between trial arms.

- A further limitation of this design is that the outcomes of the interventions may interact. While the two health domains were chosen due to the minimal overlap between behaviours, there are some plausible points of influence between the arms. For example, higher rates of pregnancy in the nutrition arm may lower haemoglobin and iron levels in these women. More generally, those receiving messages on one aspect of health may improve their health behaviours across multiple health domains, potentially diluting the effect measures of the comparison between the study arms.

- The SRH intervention aims to increase demand for contraceptives, and translating demand into raised coverage may be limited by deficiencies in the health system (supply-side issues). Supplyside issues may also influence outcomes in the nutrition group, for example, if a limited range of foods is available in the household, or area more generally

- The use of both push and pull technologies is a novel and potentially powerful approach, but their simultaneous use may make it difficult to determine the size of their independent effects.

for FSWs. ${ }^{8}$ This is due to a combination of individual barriers to uptake, such as side effects of some contraceptive methods that impact sex work and myths and misconceptions about particular methods ${ }^{910}$; interpersonal barriers, including peer norms and pressure from partners not to use contraception ${ }^{8}$ and structural barriers, such as stigmatising treatment of FSWs in the health system. ${ }^{11} 12$

Most programs established to improve the health of FSWs focus primarily on HIV and other STIs and overlook FSWs' broader reproductive needs. ${ }^{81314}$ A systematic review of SRH projects aimed at FSWs in Africa found that few provided pregnancy testing or contraceptive services other than condoms, and few specifically promoted dual method use (the concurrent use of condoms and another effective contraceptive method). ${ }^{15}$

Available data indicate that use of contraception other than condoms by FSWs in sub-Saharan Africa is variable, but generally low, ranging from around $15 \%$ to $50 \% .^{8} 16-18$ Dual method use is rarely measured, but estimated at $10 \%$ in one study in Mombasa, Kenya. ${ }^{8}$ The most popular modern method, injectable progestin, ${ }^{19}$ is prone to discontinuation and contraceptive failure due to incorrect use. ${ }^{20}{ }^{21}$ In contrast, highly effective and low maintenance long-acting reversible contraceptives (LARCs; intrauterine devices and subdermal implants) are underused, with only $7 \%$ of a sample of FSWs in Mombasa reporting ever using these methods. ${ }^{8}$

While few studies have aimed specifically to measure unintended pregnancy among FSWs, HIV prevention studies among FSWs have reported unexpectedly high rates of pregnancy, with 12 month cumulative incidence of $24 \%$ in Kenya, ${ }^{8} 23 \%$ in Madagascar, ${ }^{17} 27 \%$ in Rwanda ${ }^{22}$ and $23 \%$ in the Caribbean. ${ }^{23}$ Unintended pregnancy exposes women to significant health and social risks, such as unsafe (and often illegal) abortion, high maternal and infant morbidity and mortality for those who continue with the pregnancy ${ }^{24}$ and increased financial dependence on sex work, which in turn increases their risk of HIV, other STIs, violence and repeat pregnancies.

\section{Nutrition and nutritional status among female sex workers}

Urban food insecurity is a growing public health problem in sub-Saharan Africa and disproportionately affects poor populations, who are more exposed to nutritional risks and have less capacity to adopt effective coping strategies. ${ }^{25} \mathrm{Also}$, as a result of rapid urbanisation in low-income and middle-income countries-and associated changes in diet, leading to over-reliance on non-home prepared food that tends to be high in energy, sugar and salt and low in nutrients-undernutrition and micronutrient deficiencies are increasingly occurring alongside overnutrition, overweight and obesity. ${ }^{25-27}$ Both undernutrition and overnutrition are risk factors for poor health and mortality globally. ${ }^{28}$ In women of reproductive age, micronutrient deficiency leads to pregnancy complications and greater child and maternal morbidity and mortality. ${ }^{29}$ Anaemia, predominantly caused by iron deficiency, is endemic among women in sub-Saharan Africa; in Kenya, $36 \%$ of pregnant women and $25 \%$ of non-pregnant women are estimated to be anaemic. ${ }^{30}$

While there are no published data on malnutrition among FSWs in Kenya, lack of physical activity, chaotic lifestyles and poor diet may lead to unhealthy weight gain, at the same time contributing to their risk of micronutrient deficiency. Equally, food insecurity and hunger have been identified as key push factors for women to initiate and continue sex work. ${ }^{31}{ }^{32}$ In one study of sex workers in the Lagos metropolitan area in Nigeria, $35 \%$ of respondents had entered sex work in response to food insecurity. ${ }^{33}$

\section{mHealth interventions}

Mobile phones have the potential to effectively engage FSWs, provide information, improve knowledge and address individual and interpersonal barriers to contraception use and nutritional health. Mobile phone coverage is over $80 \%$ in Kenya ${ }^{34}$ and close to ubiquitous among FSWs, who constitute a large mobile population that relies on mobile phones for maintaining social and 
business networks (unpublished formative research from Mombasa). mHealth interventions allow for repeated, theory-driven, user-centred and low-cost exposure, which are known to be important for securing behaviour change. ${ }^{35}$

Several systematic reviews have shown that short messaging service (SMS) technology can influence health behaviour. ${ }^{36-39}$ There are, however, few randomised controlled trials (RCTs) addressing SRH behaviour through SMS. ${ }^{40-42}$ Two trials in Australia demonstrated improvements in health-seeking behaviour and sexual health outcomes, particularly among young women. ${ }^{41} 42$ Non-randomised trials and programme evaluations have also had positive findings. ${ }^{43}{ }^{44}$ With regard to nutrition, trials in USA and Iran have demonstrated the efficacy of SMS for changing eating behaviours ${ }^{45}$ and knowledge and attitudes towards iodine consumption, ${ }^{46}$ respectively. SMS interventions targeting overnutrition have also been tested, ${ }^{47-51}$ some of which were associated with significant weight reduction. ${ }^{47-49}$

Overall, however, research is failing to keep up with the rapid proliferation of mHealth interventions. ${ }^{38}$ Despite the potential gains, there remains a lack of rigorous research on SRH promotion initiatives in LMICs, ${ }^{52}$ and within the field of nutrition, the lack of rigorous data is even more pronounced. Furthermore, among populations like FSWs from low-resource settings, who tend to have lower health knowledge and fewer resources for obtaining health information, intervention impact may be greater than that observed in high-income countries, but this has yet to be demonstrated empirically.

\section{WHISPER and SHOUT interventions}

The Women's Health Intervention using SMS for Preventing Unintended Pregnancy (WHISPER) study was developed in response to the substantial SRH needs of FSWs in Kenya, the potential benefits of increasing demand for family planning among FSWs through mHealth, and the research gaps in this field. If found to be effective, mHealth for SRH could complement and increase the effectiveness of the current service packages provided for sex workers.

Similarly, the need to improve nutrition for women in poor and vulnerable settings, and the paucity of data on FSWs' nutritional health, led to the development of the SMS intervention to improve nutritional Heath OUTcomes (SHOUT). The SHOUT trial will provide important preliminary data on this underinvestigated issue and explore whether mHealth is an effective means of reducing malnutrition and anaemia in this population.

This paper presents the protocol for the WHISPER or SHOUT study. Recruitment and enrolment took place from September 2016 to May 2017, and 12-month data collection is anticipated to finish by June 2018 .

\section{METHODS AND ANALYSIS}

This is a two-arm cluster RCT, which will examine the effectiveness of parallel interventions, addressing SRH and nutrition, delivered via mobile phone among FSWs in Mombasa, Kenya. As each intervention addresses a unique set of specific issues, and the mode of delivery and level of exposure is the same, each trial arm serves as a control group for the other. ${ }^{53} 54$

\section{Study objectives and outcomes}

The overall aim is to improve the health and well-being of FSWs in resource-constrained settings. Specifically, the study will assess the effectiveness of two independent 12-month mobile phone-delivered interventions in improving: (1) SRH outcomes for FSWs receiving the WHISPER intervention, compared with women receiving a nutrition intervention and (2) nutritional status for FSWs receiving the SHOUT intervention compared with women receiving an SRH intervention.

The feasibility and acceptability of the interventions will also be assessed, using a combination of quantitative methods (structured questionnaires at 6 and 12 months) and qualitative methods (in-depth interviews at 12 months).

\section{WHISPER}

The primary study outcome for WHISPER is the incidence of unintended pregnancy over 12 months of follow-up. Unintended pregnancy is defined as pregnancy that is mistimed, unplanned or unwanted at the time of conception. ${ }^{55}$ Women in the study are asked about their pregnancy intentions for the forthcoming 6 months at enrolment and at the 6-month and 12-month visits. A psychometrically-validated six-item questionnaire (London Measure of Unintended Pregnancy) will also be used to score pregnancy intention when pregnancy occurs during study follow-up. ${ }^{56}$ Pregnancy events are defined as either a positive result on urine pregnancy screening at the 6-month or 12-month visits (or at unscheduled visits at the study clinics, should these occur) or a self-reported pregnancy that occurs between study visits (captured by the follow-up questionnaire).

Secondary outcomes are being measured at baseline and 6 and 12 months and include incidence of HIV and syphilis measured by point-of-care testing. Self-reported secondary outcomes, assessed by structured questionnaire, include SRH knowledge, behaviour (prevalence of LARC and dual method use) and service utilisation.

\section{SHOUT}

The primary study outcome for SHOUT is the prevalence of anaemia at 12 months of follow-up. This outcome is measured using a certified laboratory-based haematology machine at baseline and 12 months and is defined as haemoglobin $(\mathrm{Hb})$ level below $12.0 \mathrm{~g} / \mathrm{dL}$, consistent with the WHO definition. ${ }^{30}$ A point-of-care haemoglobinometer will also be used to enable immediate management of anaemia, but the laboratory measure will be used to calculate the primary outcome. 


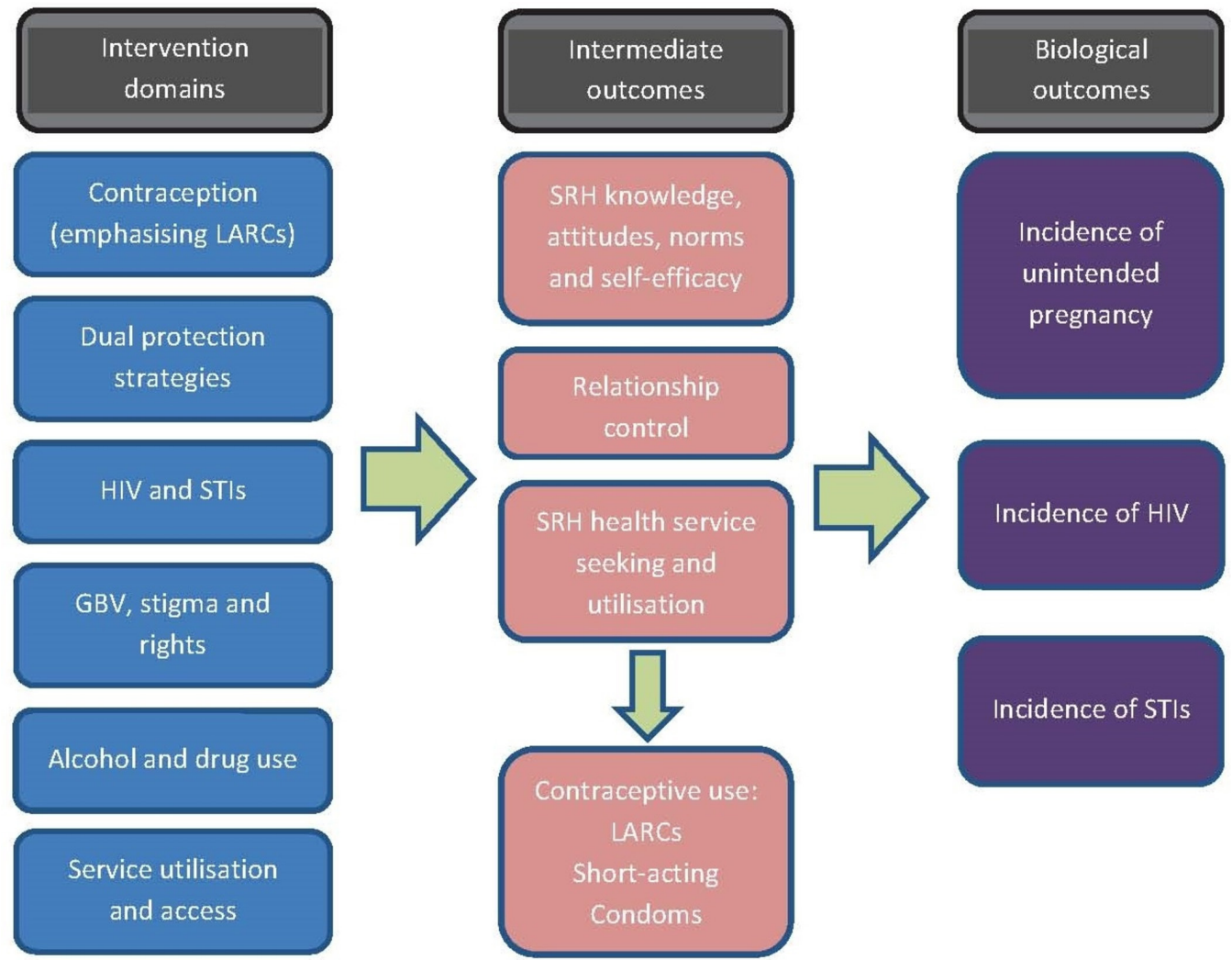

Figure 1 Logic model for the SRH intervention (WHISPER). LARCs, long-acting reversible contraceptives; SRH, sexual and reproductive health; STIs, sexually transmitted infections; GBV, gender-based violence.

Secondary outcomes include mean $\mathrm{Hb}$ levels at 12 months, prevalence of malnutrition (measured by body mass index and mid-upper arm circumference (MUAC) at baseline and 6 and 12 months) and self-reported nutritional knowledge and behaviour (assessed by structured questionnaire at baseline and 6 and 12 months).

\section{Study intervention}

Development of WHISPER or SHOUT study interventions

Changes in attitudes and behaviours are most likely to occur when communication interventions are theory-driven, interactive and follow best practice in design and implementation. ${ }^{57}$ Furthermore, sustainable positive behaviour change among individuals and communities occurs as a process, supported by carefully designed interventions, and not as a single event. ${ }^{5859}$

The WHISPER and SHOUT interventions were therefore developed following a predefined protocol and in a systematic and participatory manner, ensuring that the intervention was grounded in evidence, was relevant and acceptable to the study population and followed a logical process of behaviour change based on staged and social cognitive theoretical approaches. ${ }^{60}$ An extensive review of the literature and consideration of health behaviour change theory informed the development of logic models for both study arms (figures 1 and 2) and the subsequent drafting of messages. Participatory and user-centred design and testing of the mobile phone intervention ${ }^{61-63}$ was conducted with the target population in 4 informal consultative meetings, 12 formal workshops and 24 usability testing interviews. FSWs were involved in identifying important issues that affect them, developing messages and role model stories, reviewing and refining the content and suggesting changes to the intervention architecture.

\section{Structure and delivery of the intervention}

Both the SRH and nutrition intervention consist of SMS (text) messages sent to participants two to three times per week for 12 months. The intervention includes the following components:

- 'push' texts covering the main content domains, providing simple information, motivational messages and strategies to prompt action;

- 'role model stories' about FSWs modelling healthy social norms and sent in 4-5 instalments in 1 month (alternating months with stand-alone messages) and

- a 'pull' or on-demand menu that participants can access by texting into the system to obtain more information on high-priority topics and local services.

The intervention is being delivered by the VOTO mobile (https://www.votomobile.org/) online platform. All messages are loaded onto the system in a predetermined order and sent automatically to each cluster of participants at the scheduled time (calculated from each cluster's start date). Participants are able to text assigned codes at any time during the study to access the pull messages, with SMS 


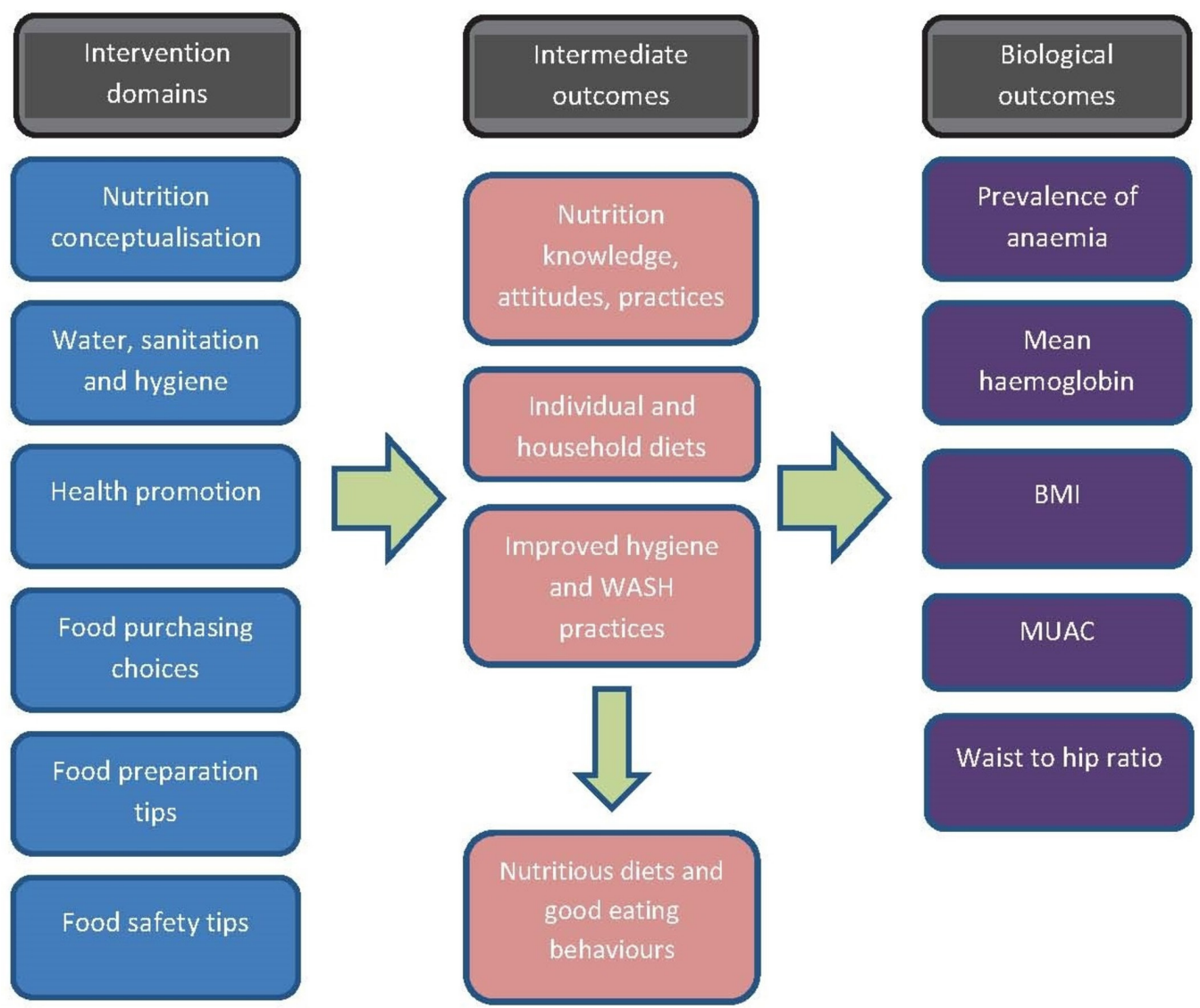

Figure 2 Logic model for the nutrition intervention (SHOUT). BMI, body mass index; MUAC, middle-upper arm circumference.

costs incurred by the study (no charge for participants). Content is only accessible to enrolled participants in the relevant intervention group, so that those in the nutrition arm cannot directly access SRH messages and vice versa.

Table 1 presents a summary of the structure of the interventions, showing that they are essentially equivalent in all structural components except the on-demand system. This discrepancy is due to additional pull messages in WHISPER that provide information about SRH services, allowing participants to link in with local sex worker-sensitive providers. This was deemed important on ethical grounds given the sensitive content of the WHISPER intervention and because promotion of contraception can only impact pregnancy

Table 1 Number, length and timing of messages for each study arm

\begin{tabular}{|c|c|c|c|}
\hline Message type & Number of messages & Length & Timing \\
\hline Push messages & 82 (both arms) & $\begin{array}{l}\text { Up to } 160 \text { characters ( } 1 \text { SMS } \\
\text { 'screen')* }\end{array}$ & $\begin{array}{l}\text { Three per week (Monday, } \\
\text { Wednesday and Saturday } \\
\text { mornings) during months } 1,3 \text {, } \\
5,7,9,11,13^{\dagger}\end{array}$ \\
\hline $\begin{array}{l}\text { Interview reminders and study } \\
\text { information (push messages) }\end{array}$ & 7 (both arms) & $\begin{array}{l}160-480 \text { characters }(1-3 \\
\text { screens) }\end{array}$ & Months $6,7,13$ \\
\hline $\begin{array}{l}\text { 'Alerts': push messages linking } \\
\text { participants to the pull system }\end{array}$ & 19 (SRH); 18 (nutrition) & $\begin{array}{l}160-320 \text { characters }(1-2 \\
\text { screens) }\end{array}$ & \\
\hline Pull (on-demand) messages & 80 (SRH); 49 (nutrition) & $\begin{array}{l}160-640 \text { characters }(1-4 \\
\text { screens) }\end{array}$ & User determined \\
\hline
\end{tabular}

*Messages are charged per 160 characters (one screen). Messages longer than this will appear as one long SMS on most phones, but may be split into multiple messages on older phones.

†There are 13 blocks of 4 weeks each, making up 12 calendar months in total.

$\mathrm{SMS}$, short messaging service; $\mathrm{SRH}$, sexual and reproductive health. 
rates if delivered alongside accessible services that provide the methods in question. In contrast, the promotion of healthy eating does not require an intermediate service utilisation step to result in improved nutritional outcomes. Furthermore, low-cost or free nutrition services are not widely available in Mombasa, and screening asymptomatic non-pregnant women for anaemia is rarely practised, so we were unable to reliably list relevant services for SHOUT.

\section{Setting and participants}

Eligible women were recruited from mapped sex work venues by well-established peer outreach workers. Sex work venues consist of fixed-site businesses including nightclubs, bars, brothels and hotels as well as public spaces such as street corners and beaches, where sex is known to be bought and sold. The study is being conducted in two areas of Mombasa, a major economic centre in Kenya and East Africa, with busy port, rail and industrial enterprises. The local implementing partner, International Centre for Reproductive Health (ICRH; a WHO Collaborating Centre for Reproductive Health in Mombasa) has a research track record in the area dating back to 2002, including many trials and other prospective intervention studies with FSWs ${ }^{64-66}$ and numerous non-interventional studies. ${ }^{67-70}$

\section{Inclusion and exclusion criteria}

To be eligible for participation, women had to: (1) be aged 16 to 35 years; (2) report having engaged in sex work (received money in exchange for sex) at the site of recruitment in the last 6 months; (3) not be pregnant or planning pregnancy within 12 months; (4) reside within the study area for the duration of the study; (5) have a personal mobile phone with Safaricom or Airtel subscription, and be willing to provide the phone number to the researchers to receive the intervention messages; (6) report being SMS literate (ie, able to read text messages in English); (7) be willing to return for follow-up after 6 and 12 months; (8) be willing to provide contact information (eg, home address) to enable contact or a visit from a member of the research team in the community to remind them to attend follow-up visits and (9) be able and willing to give written informed consent for enrolment in the study.

Exclusion criteria included participation in another mHealth intervention study or in the formative research for this study and having a medical or non-medical condition detected through screening that hinders study participation, as confirmed by the local principal investigator.

\section{Randomisation and sampling strategy}

ICRH and collaborators conducted a two-stage geographic mapping and enumeration exercise of FSWs in 2014, estimating that 11777 (range 9265-14290) FSWs operate from 1053 venues across four subcounties of Mombasa County. ${ }^{62}$

We are recruiting FSW from two of these areas: Changamwe (where an estimated 3435 FSWs work from 285 venues) and Kisauni (where an estimated 5081 FSWs work from 475 venues). ${ }^{62}$

A two-stage sampling process was adopted, drawing on this sampling frame. At the first stage, FSW venues were selected with a probability proportionate to the enumerated size of the sex worker population at the venue and randomised to either the WHISPER or SHOUT arm. Each venue was considered to be a separate cluster. At the second stage, 10 FSWs were consecutively selected from each venue, based on an estimated mean cluster size of $11{ }^{62}$ At venues where less than $10 \mathrm{FSWs}$ were active, all FSWs from the selected venue were invited to participate in the study. Sampling of FSW venues continued until the required sample size was achieved.

Cluster randomisation (allocation of each cluster to either WHISPER or SHOUT) was done centrally by the statistician and prior to recruitment commencing, involving an equal number of clusters in both arms. The participants and study team were blinded to allocation until after cluster enrolment was completed and all baseline questionnaires had been administered for that cluster.

Overall, our approach aimed for optimal (as random as possible) sampling from the FSW population, while minimising the potential intervention dilution effects of message sharing that might occur with individual-level randomisation.

\section{Recruitment and enrolment}

Community mobilisers and peer educators employed by the study applied minimum prescreening criteria at the site of recruitment to identify potential study participants. Potentially eligible women received a card with a unique, anonymous referral card number (made up of cluster number and sequential participant number for that cluster). They were asked to attend the nearest study clinic for full eligibility screening, consenting and enrolment within the next 3 days. The study clinics are open 5 days a week and are embedded in existing facilities: an ICRH-run sex-worker drop-in centre in Kisauni and a municipal community health centre in Changamwe. The number of sex workers approached and referral cards disbursed at each cluster was documented as well as reasons for ineligibility at prescreening.

Volunteers had to bring their referral card number with them to undergo the screening process to ensure that only volunteers selected by the random sampling process were enrolled. Women who consented to participate and successfully completed full screening had their study identification number assigned and were then formally enrolled.

\section{Data collection}

Study visits were conducted at enrolment and will be repeated after 6 and 12 months at the study clinics. All study staff, including clinicians, research assistants, community mobilisers and peer educators were trained in the study procedures during a 5-day workshop and undertake additional targeted sessions where required. 
Table 2 Schedule of assessments for the WHISPER or SHOUT trial

\begin{tabular}{lc}
\hline Assessments & Month 0 \\
\hline Structured questionnaire & Month 6 \\
Clinical examination & Month 12 \\
\hline Urine pregnancy testing & $\checkmark$ \\
Point-of-care HIV and syphilis testing (including pretest and post-test counselling) & $\checkmark$ \\
Haemoglobin measured by haemoglobinometer & $\checkmark$ \\
Full blood count & $\checkmark$ \\
In-depth interviews (subgroup of participants from sexual and reproductive health arm) & $\checkmark$ \\
\hline
\end{tabular}

Enrolment procedures include collecting informed consent, administering the baseline questionnaire and performing clinical examination, urine pregnancy test, point-of-care blood tests for HIV, syphilis and Hb level and venepuncture for full blood count (haemogram: $\mathrm{Hb}$, haematocrit, mean cell volume and white blood count). Follow-up assessments are presented in table 2.

Questionnaires were developed in English and translated into Kiswahili and were based on previously validated measurement tools wherever possible. The questionnaire captures detailed sociodemographic information including education, literacy, employment, income, family and living circumstances, health and illness and sex work history. SRH enquiries cover previous pregnancy(ies); 6-month pregnancy intention; contraception and condom use (including dual protection); reasons for discontinuation or non-use of contraception; contraceptive self-efficacy; SRH seeking and service utilisation; sexual risk behaviours; SRH knowledge and attitudes and relationship control and joint-decision-making with non-paying emotional partners. Participants are asked about whether they have recent STI-related symptoms, which are classified and treated as per syndromic STI guidelines. ${ }^{71}$ Nutrition questions gather data on nutrition-related health seeking and service utilisation; food and hygiene-related knowledge and behaviours; dietary intake and preparation and food purchasing.

Clinical examination is performed by study clinicians and includes STI syndromic management and anthropometric measurements to assess nutritional status (ie, weight, height, waist circumference, hip circumference and MUAC).

The results of rapid diagnostic tests are communicated to the participants at the time of the visit. Participants newly diagnosed with HIV are referred for treatment at specialised centres. Those with positive syphilis rapid test are treated with benzathine penicillin at the study visit. Participants with anaemia receive 1 month of iron and folic acid supplementation and a referral to a health facility for follow-up.

Following enrolment, each participant was registered in the VOTO online platform. No identifying details apart from mobile phone number were entered. Intervention allocation was performed by data management staff independent of the study team and occurred after enrolment was completed for each cluster. The intervention commenced simultaneously for all participants of one cluster in the week following completed cluster-level enrolment.

\section{Data analyses}

Analysis of primary endpoints

Primary analysis for WHISPER and SHOUT will compare the primary endpoints (unintended pregnancy incidence and anaemia prevalence, respectively) between groups at 12 months. The data analysis team will be blinded to the allocation of participants. Given the interval-censored nature of unintended pregnancy incidence, discrete-time survival models using generalised linear modelling will be used to compare unintended pregnancy incidence between the two trial arms. Comparison of differences in prevalence of anaemia between study arms will be undertaken using multilevel generalised linear modelling.

These analyses will, where appropriate, provide estimates with robust SEs for FSW venue clustering. Standardised probability weighting will be applied in population-averaged analyses to account for any sampling bias where achieved sample cluster sizes vary. Also, where randomisation is not effective in removing allocation bias, adjusted models will be specified. In all analyses, associations will be considered statistically significant at the $5 \%$ level.

\section{Sample size}

The sample size was calculated to obtain sufficient power to examine the effects of the SRH intervention. Based on a 12-month incidence of unintended pregnancy of $24 \%$ in the control group (data from previous research in the study population), ${ }^{8}$ enrolment of 860 participants from a minimum of 86 FSW venues would enable detection of a relative reduction in annual unintended pregnancy incidence of $37 \%$ (hazard risk $=0.63$ ), at $80 \%$ power and $5 \%$ significance level. This estimate was adjusted for an expected $10 \%$ attrition rate (based on previous experience with this population ${ }^{8}$ ) and an estimated inflation in $\mathrm{SE}$ due to cluster randomisation (design effect $=1.18$; estimated intracluster correlation coefficient $=0.02$, with a cluster size of 10). ${ }^{72}$ 
Sample size could not be reliably calculated for the SHOUT trial as estimates of anaemia in the local sex work population are not available. However, if we assume a prevalence of $25 \%$ (as per the national estimate for non-pregnant women ${ }^{30}$ ), the calculated sample size of 860 would allow for detection of an approximate $42 \%$ reduction in odds of anaemia prevalence, with inflation in SE and attrition rate adjustments applied as above.

\section{ETHICS AND DISSEMINATION}

Written informed consent has been obtained from every study participant. Women aged 16 and 17 years are considered mature minors and able to consent without involving parents and guardians, due to the sensitive nature of the subject matter. Informed consent forms are available in both Kiswahili and English and describe the purpose of the study, the procedures to be followed and the risks and benefits of participation. Most importantly, we are aware of the risk that others seeing the messages may infer that the participant is a sex worker. It is important to mitigate this risk of unintentional disclosure of sex work status and consequent stigma or other adverse events for participants. ${ }^{73}$ Participants have been counselled about the need for care to be taken to ensure that others do not have access to their phones. The study team also asks that participants report instances of unintentional disclosure. Such reporting allows us to provide support to the women concerned and, through understanding the events surrounding the case, try to find ways to avoid future occurrences.

Ethical approvals have been obtained for protocol version 1.2, dated 22 August 2016 from the Monash University Human Research Ethics Committee (MUHREC-CF16/1552-2016000812) and the Kenyatta National Hospital, University of Nairobi Ethics and Research Committee (KNH-UoN ERC-KNH-ERC/RR/493).

Several dissemination activities are planned, including to health workers and the district office, Kenyan policymakers, at local and international conferences and in academic publications.

\section{Potential impact and significance}

The WHISPER study responds to the pressing need for effective and evidence-informed interventions to prevent unintended pregnancies among vulnerable women in resource-constrained settings. Persistent unmet need for family planning contributed to inadequate progress towards reducing child mortality and improving maternal health (Millennium Development Goals 4 and 5) and to the need for the 2010 Global Strategy for Women's and Children's Health. ${ }^{7}$ The ability to decide on the number and timing of children, free from coercion and violence, is a fundamental human right. During the 2012 London Summit on Family Planning, ${ }^{74}$ the Kenyan Government committed to increasing funding and contraception coverage by 2015 . Despite this supply-side commitment, questions remain about the optimum mechanism to increase demand, particularly among marginalised populations. The WHISPER trial will assess an innovative, sustainable and scalable approach to address some of these gaps.

Study outcomes will be used to inform future policies and public health interventions aimed at increasing uptake of family planning as well as other SRH services, among vulnerable populations in a range of similar settings, particularly in LMICs. The rigorous research design and extensive local experience of our team will support advocacy and guide mobile technology services for health promotion as outlined in the 2010 Kenyan Government's Family Planning Guidelines. ${ }^{75}$ If found to be effective, this intervention could be sustainably scaled up across East Africa.

With regard to nutritional health, there are renewed efforts to design and implement action-oriented research, but the role of mHealth in these initiatives remains uncertain. ${ }^{76}$ The SHOUT study will go some way towards clarifying whether SMS technology is a useful way of responding to a long-recognised, but poorly characterised and neglected problem. Indeed, if shown to be effective in this trial, mHealth could be incorporated into broader nutrition strategies for FSWs in Africa and elsewhere and has the potential to be scaled up for use with other women at a population level.

The design mitigates biases from unequal attention in trial groups, positive expectations and non-blinding. ${ }^{5354}$ The innovative approach described herein has never been used to prevent unintended pregnancies or improve nutrition among key risk populations in LMICs. A similar methodology in which the effects of SRH text messages were compared with a control SMS intervention was adopted in Australia with a sun safety control group,${ }^{41}$ and in USA with a nutrition control group, ${ }^{44}$ although the latter did not measure nutrition outcomes, unlike our trial. This trial will thus set standards for the mHealth field in LMICs, for both interventions and trial methodology. Lessons learnt about optimising SMS technology for both WHISPER and SHOUT could have considerable impact in Kenya and similar countries, for a range of health priorities outside of those examined here. $^{69}$

In conclusion, FSWs constitute a large, vulnerable, hardto-reach population that could be amenable to mHealth interventions given their high mobile phone coverage and utilisation. FSWs are at considerable risk of unintended pregnancies and could benefit markedly from interventions that increase uptake of contraception, with an emphasis on long-acting reversible technologies and dual method use. Similarly, the nutrition arm of the trial responds to the paucity of quality data on rates of malnutrition among sex workers and of effective interventions to improve their nutritional status and metabolic health.

A cluster RCT with an equal-attention control and objective biological primary endpoints (unintended pregnancy and anaemia) is the most robust study design to test the effectiveness of the planned 
interventions. It is anticipated that the trial will contribute important evidence to mHealth initiatives among vulnerable populations and provide useful methodological insights into the use of reciprocal control groups within such trials.

\section{Author affiliations}

${ }^{1}$ Burnet Institute, Melbourne, Australia

${ }^{2}$ Department of Epidemiology and Preventive Medicine, Monash University,

Melbourne, Australia

${ }^{3}$ International Centre for Reproductive Health (ICRH), Mombasa, Kenya

${ }^{4}$ University of Nairobi, Mombasa, Kenya

${ }^{5}$ Department of Obstetrics and Gynaecology, International Centre for Reproductive Health (ICRH), Ghent University, Ghent, Belgium

${ }^{6}$ Wits Reproductive Health and HIV Institute, University of the Witwatersrand,

Johannesburg, South Africa

${ }^{7}$ Aga Khan University, Nairobi, Kenya

${ }^{8}$ Judith Lumley Centre, La Trobe University, Melbourne, Australia

${ }^{9}$ University of San Francisco, San Francisco, California, USA

Acknowledgements The authors gratefully acknowledge the contribution to this work of funding from the Victorian Operational Infrastructure Support Programme received by the Burnet Institute.

Contributors The following authors were investigators on the trial from the outset: FHA, CM, PG, MSCL, MC, WJ, MT, ML, LC-T, MS, PAA, MH, KL SL. GM more recently joined the trial site in Kenya, leading work in that site. The paper draws from the trial protocol, which was written collectively, under the leadership of the Principal Investigator SL. The final version of the paper was read and approved by all authors.

Funding Australia's National Health and Medical Research Council (NHMRC) provided funding for Project Grant GNT 1087006, Career Development Fellowships for SL and MS, and a Postgraduate Scholarship for FHA. The sponsor did not have any contribution to the study design, data collection, management, analysis or interpretation.

Competing interests None declared.

Patient consent Obtained.

Ethics approval Monash University (MUHREC) in Australia and Kenyatta National Hospital Ethics and research committee in Kenya.

Provenance and peer review Not commissioned; externally peer reviewed.

Data sharing statement Data presented in this paper are available from the corresponding author on request.

Open Access This is an Open Access article distributed in accordance with the Creative Commons Attribution Non Commercial (CC BY-NC 4.0) license, which permits others to distribute, remix, adapt, build upon this work non-commercially, and license their derivative works on different terms, provided the original work is properly cited and the use is non-commercial. See: http://creativecommons.org/ licenses/by-nc/4.0/

(C) Article author(s) (or their employer(s) unless otherwise stated in the text of the article) 2017. All rights reserved. No commercial use is permitted unless otherwise expressly granted.

\section{REFERENCES}

1. Vandepitte J, Lyerla R, Dallabetta G, et al. Estimates of the number of female sex workers in different regions of the world. Sex Transm Infect 2006;82 Suppl 3(Suppl 3:iii18):iii18-iii25.

2. Odek WO, Githuka GN, Avery L, et al. Estimating the size of the female sex worker population in Kenya to inform HIV prevention programming. PLoS One 2014;9:e89180.

3. The Global Fund. Key Populations Action Plan 2014-2017. Geneva: the Global Fund to fight AIDS, tuberculosis and malaria. 2013.

4. Kerrigan D, Kennedy CE, Morgan-Thomas R, et al. A community empowerment approach to the HIV response among sex workers: effectiveness, challenges, and considerations for implementation and scale-up. Lancet 2015;385:172-85.

5. Kyalo J, Jurczynska K, Kundu F. ImpactNOW application in Kenya: policy brief. Washington: Health Policy Project \& National Council for Population and Development, 2015.
6. Ross J, Stover J. Use of modern contraception increases when more methods become available: analysis of evidence from 1982-2009. Glob Health Sci Pract 2013;1:203-12.

7. Temmerman M, Khosla R, Bhutta ZA, et al. Towards a new global strategy for Women's, Children's and Adolescents' Health. BMJ 2015;351:h4414.

8. Luchters S, Bosire W, Feng A, et al. "A Baby Was an Added Burden": Predictors and Consequences of Unintended Pregnancies for Female Sex Workers in Mombasa, Kenya: A Mixed-Methods Study. PLoS One 2016;11:e0162871.

9. Khan MR, Turner AN, Pettifor A, et al. Unmet need for contraception among sex workers in Madagascar. Contraception 2009;79:221-7.

10. Sutherland EG, Alaii J, Tsui S, et al. Contraceptive needs of female sex workers in Kenya - a cross-sectional study. Eur $\mathrm{J}$ Contracept Reprod Health Care 2011;16:173-82.

11. Scorgie F, Nakato D, Harper E, et al. 'We are despised in the hospitals': sex workers' experiences of accessing health care in four African countries. Cult Health Sex 2013;15:450-65.

12. Mannava P, Durrant K, Fisher J, et al. Attitudes and behaviours of maternal health care providers in interactions with clients: a systematic review. Global Health 2015;11:36.

13. Schwartz S, Papworth E, Thiam-Niangoin M, et al. An urgent need for integration of family planning services into HIV care: the high burden of unplanned pregnancy, termination of pregnancy, and limited contraception use among female sex workers in Côte D'ivoire. $J$ Acquir Immune Defic Syndr 2015;68:S91-S98.

14. Weldegebreal R, Melaku YA, Alemayehu M, et al. Unintended pregnancy among female sex workers in Mekelle city, northern Ethiopia: a cross-sectional study. BMC Public Health 2015;15:40.

15. Dhana A, Luchters S, Moore L, et al. Systematic review of facilitybased sexual and reproductive health services for female sex workers in Africa. Global Health 2014;10:46-

16. Erickson M, Goldenberg SM, Ajok M, et al. Structural determinants of dual contraceptive use among female sex workers in Gulu, northern Uganda. Int J Gynaecol Obstet 2015;131:91-5.

17. Feldblum PJ, Nasution MD, Hoke TH, et al. Pregnancy among sex workers participating in a condom intervention trial highlights the need for dual protection. Contraception 2007;76:105-10.

18. McClelland RS, Richardson BA, Cherutich P, et al. A 15-year study of the impact of community antiretroviral therapy coverage on HIV incidence in kenyan female sex workers. AIDS 2015;29:2279-86.

19. Darroch JE, Singh S. Trends in contraceptive need and use in developing countries in 2003, 2008, and 2012: an analysis of national surveys. Lancet 2013;381:1756-62.

20. Baumgartner JN, Morroni C, Mlobeli RD, et al. Timeliness of contraceptive reinjections in South Africa and its relation to unintentional discontinuation. Int Fam Plan Perspect 2007:33:066-74.

21. Smit JA, Beksinska ME. Hormonal contraceptive continuation and switching in South Africa: implications for evaluating the association of injectable hormonal contraceptive use and HIV. J Acquir Immune Defic Syndr 2013;62:363-5.

22. Braunstein SL, Ingabire CM, Kestelyn E, et al. High human immunodeficiency virus incidence in a cohort of rwandan female sex workers. Sex Transm Dis 2011;38:385-94.

23. Deschamps MM, Metch B, Morgan CA, et al. HVTN 907 Study Team. Feasibility of identifying a female sex worker cohort at high risk of HIV infection in the Caribbean for HIV vaccine efficacy trials: longitudinal results of HVTN 907. J Acquir Immune Defic Syndr 2016;71:70-7.

24. Singh S, Darroch JE, Ashford LS. Adding it up: the costs and benefits of investing in sexual and reproductive health. New York: Guttmacher Institute, 2014.

25. Kimani-Murage EW, Schofield L, Wekesah F, et al. Vulnerability to food insecurity in urban slums: experiences from Nairobi, Kenya. $J$ Urban Health 2014:91:1098-113.

26. van 't Riet $\mathrm{H}$, den Hartog AP, Hooftman DA, et al. Determinants of non-home-prepared food consumption in two low-income areas in Nairobi. Nutrition 2003;19(11-12):1006-12.

27. van't Riet $H$, den Hartog AP, van Staveren WA. Non-home prepared foods: contribution to energy and nutrient intake of consumers living in two low-income areas in Nairobi. Public Health Nutr 2002;5:515-22.

28. Oldewage-Theron $\mathrm{WH}$, Kruger R, Egal AA. Socio-Economic variables and Nutrient Adequacy of Women in the Vaal Region of South Africa. Ecol Food Nutr 2014;53:514-27.

29. United Nations Children's Fund, United Nations University, World Health Organization. Iron deficiency anaemia, assessment, control and prevention: a guide for programme managers. 2010.

30. World Health Organization. The global prevalence of anaemia in 2011. Geneva, 2015. 
31. Fielding-Miller R, Mnisi Z, Adams D, et al. "There is hunger in my community": a qualitative study of food security as a cyclical force in sex work in Swaziland. BMC Public Health 2014;14:79.

32. Kunnuji M. Basic deprivation and involvement in risky sexual behaviour among out-of-school young people in a Lagos slum. Cult Health Sex 2014;16:727-40.

33. Oyefara JL. Food insecurity, HIV/AIDS pandemic and sexual behaviour of female commercial sex workers in Lagos metropolis, Nigeria. Sahara J 2007;4:626-35.

34. International Telecommunication Union. Information and Communication Technology (ICT) Statistics. 2016 http://www.itu.int/ en/ITU-D/Statistics/Pages/stat/default.aspx (accessed 10 Sep 2016)

35. Mwaikambo L, Speizer IS, Schurmann A, et al. What works in family planning interventions: a systematic review. Stud Fam Plann 2011:42:67-82

36. Cole-Lewis $\mathrm{H}$, Kershaw T. Text messaging as a tool for behavior change in disease prevention and management. Epidemiol Rev 2010;32:56-69.

37. Fjeldsoe BS, Marshall AL, Miller YD. Behavior change interventions delivered by mobile telephone short-message service. Am J Prev Med 2009;36:165-73.

38. Free C, Phillips G, Watson L, et al. The effectiveness of mobile-health technologies to improve health care service delivery processes: a systematic review and meta-analysis. PLoS Med 2013;10:e1001363.

39. Armanasco AA, Miller YD, Fjeldsoe BS, et al. Preventive Health Behavior Change text message interventions: a Meta-analysis. Am J Prev Med. In Press. 2017;52:391-402.

40. Tsur L, Kozer E, Berkovitch M. The effect of drug consultation center guidance on contraceptive use among women using isotretinoin: a randomized, controlled study. J Womens Health 2008;17:579-84.

41. Gold J, Aitken CK, Dixon HG, et al. A randomised controlled trial using mobile advertising to promote safer sex and sun safety to young people. Health Educ Res 2011;26:782-94.

42. Lim MS, Hocking JS, Aitken CK, et al. Impact of text and email messaging on the sexual health of young people: a randomised controlled trial. J Epidemiol Community Health 2012;66:69-74.

43. L'Engle KL, Vahdat HL, Ndakidemi E, et al. Evaluating feasibility, reach and potential impact of a text message family planning information service in Tanzania. Contraception 2013;87:251-6.

44. Juzang I, Fortune T, Black S, et al. A pilot programme using mobile phones for HIV prevention. J Telemed Telecare 2011;17:150-3.

45. Brown ON, O'Connor LE, Savaiano D. Mobile MyPlate: a pilot study using text messaging to provide nutrition education and promote better dietary choices in college students. J Am Coll Health 2014;62:320-7.

46. Mehran L, Nazeri P, Delshad H, et al. Does a text messaging intervention improve knowledge, attitudes and practice regarding iodine deficiency and iodized salt consumption? Public Health Nutr 2012;15:2320-5.

47. Haapala I, Barengo NC, Biggs S, et al. Weight loss by mobile phone: a 1-year effectiveness study. Public Health Nutr 2009;12:2382-91.

48. Lombard C, Deeks A, Jolley D, et al. A low intensity, community based lifestyle programme to prevent weight gain in women with young children: cluster randomised controlled trial. BMJ 2010;341:c3215.

49. Joo NS, Kim BT. Mobile phone short message service messaging for behaviour modification in a community-based weight control programme in Korea. J Telemed Telecare 2007;13:416-20.

50. Patrick K, Raab F, Adams MA, et al. A text message-based intervention for weight loss: randomized controlled trial. $J$ Med Internet Res 2009;11:e1.

51. Shapiro JR, Bauer S, Hamer RM, et al. Use of text messaging for monitoring sugar-sweetened beverages, physical activity, and screen time in children: a pilot study. J Nutr Educ Behav 2008;40:385-91.

52. Lim MS, Hocking JS, Hellard ME, et al. SMS STI: a review of the uses of mobile phone text messaging in sexual health. Int J STD AIDS 2008;19:287-90.

53. Hartinger SM, Lanata CF, Hattendorf J, et al. Improving household air, drinking water and hygiene in rural Peru: a communityrandomized-controlled trial of an integrated environmental homebased intervention package to improve child health. Int J Epidemiol 2016;45:dyw242.

54. Hartinger SM, Lanata CF, Hattendorf J, et al. Impact of a child stimulation intervention on early child development in rural Peru: a cluster randomised trial using a reciprocal control design. $J$ Epidemiol Community Health 2017;71:217-24.

55. US Centers for Disease Control and Prevention. Unintended Pregnancy Prevention 2015 https://www.cdc.gov/reproductivehealth/ unintendedpregnancy/ (accessed 22 Dec 2016).

56. Hall J, Barrett G, Mbwana N, et al. Understanding pregnancy planning in a low-income country setting: validation of the London measure of unplanned pregnancy in Malawi. BMC Pregnancy Childbirth 2013;13:200.

57. Noar SM, Palmgreen P, Chabot M, et al. A 10-year systematic review of HIV/AIDS mass communication campaigns: Have we made progress? J Health Commun 2009;14:15-42.

58. WIC Programme. WIC programme nutrition education guidance, 2006.

59. California WIC Programme. How to evaluate a WIC Nutrition education program, 2002

60. US Department of Health and Human Services. Making health communication programs work. Washington, DC: US Department of Health and Human Services, 2004.

61. Zhang D, Adipat B, Challenges AB. Challenges, methodologies, and issues in the Usability Testing of Mobile applications. Int $J$ Hum Comput Interact 2005;18:293-308.

62. Cheuk E, Becker M, Mishra S, et al. Understanding female sex workers' Early HIV Risk and the Implications for HIV Epidemic Control (Transitions Study): Mapping and Estimating the Population Size of Female Sex Workers in Mombasa, Kenya and Dnipropetrovsk:Ukraine. 24th Annual Canadian Conference on HIV/ AIDS Research. Can J Infect Dis Med Microbiol 2015;78B.

63. Yardley L, Morrison L, Bradbury K, et al. The person-based approach to intervention development: application to digital health-related behavior change interventions. J Med Internet Res 2015;17:e30.

64. Luchters S, Chersich MF, Jao I, et al. Acceptability of the diaphragm in Mombasa Kenya: a 6-month prospective study. Eur J Contracept Reprod Health Care 2007;12:345-53.

65. Thomsen SC, Ombidi W, Toroitich-Ruto C, et al. A prospective study assessing the effects of introducing the female condom in a sex worker population in Mombasa, Kenya. Sex Transm Infect 2006;82:397-402.

66. L'Engle KL, Mwarogo P, Kingola N, et al. A randomized controlled trial of a brief intervention to reduce alcohol use among female sex workers in Mombasa, Kenya. J Acquir Immune Defic Syndr 2014:67:446-53.

67. Chersich MF, Luchters SM, Malonza IM, et al. Heavy episodic drinking among kenyan female sex workers is associated with unsafe sex, sexual violence and sexually transmitted infections. Int J STD AIDS 2007;18:764-9.

68. Luchters S, Chersich MF, Rinyiru A, et al. Impact of five years of peermediated interventions on sexual behavior and sexually transmitted infections among female sex workers in Mombasa, Kenya. BMC Public Health 2008;8:143.

69. Luchters S, Richter ML, Bosire W, et al. The contribution of emotional partners to sexual risk taking and violence among female sex workers in Mombasa, Kenya: a cohort study. PLoS One 2013;8:e68855

70. Pack AP, L'engle K, Mwarogo P, et al. Intimate partner violence against female sex workers in Mombasa, Kenya. Cult Health Sex 2013

71. World Health Organization. Guidelines for the management of sexually transmitted infections. 2004.

72. Cai Y, Shi R, Shen T, et al. A study of HIV/AIDS related knowledge, attitude and behaviors among female sex workers in Shanghai China. BMC Public Health 2010;10:377.

73. Rodrigues R, Poongulali S, Balaji K, et al. 'The phone reminder is important, but will others get to know about my illness?' Patient perceptions of an mHealth antiretroviral treatment support intervention in the HIVIND trial in South India. BMJ Open 2015;5:e007574.

74. Carr B, Gates MF, Mitchell A, et al. Giving women the power to plan their families. Lancet 2012;380:80-2.

75. National Family Planning guidelines for Service Providers. Kenya: Reproductive Health Division, 2010.

76. Beratarrechea A, Moyano D, Irazola V, et al. mHealth Interventions to Counter Noncommunicable Diseases in developing countries: still an Uncertain Promise. Cardiol Clin 2017;35:13-30. 